International Mathematical Forum, 1, 2006, no. 16, 769-795

\title{
Separation of Convolved Mixtures in Frequency Domain ICA
}

\author{
A. Ciaramella and R. Tagliaferri \\ Dept. of Mathematics anf Informatics \\ via Ponte don Melillo, I-84084, Fisciano (SA) \\ Italy \\ M. Funaro \\ Dept. of Physics \\ via S. Allende, I-84081, Fisciano (SA) \\ Italy
}

\begin{abstract}
In this paper, a novel approach to solve the permutation indeterminacy in the separation of convolved mixtures in frequency domain is proposed. A fixed-point algorithm in complex domain is used to separate the signals in each frequency bin. These are obtained applying a Short Time Fourier Transform on a set of fixed frames. To solve the ambiguity of the amplitude dilation, a simple method is proposed. The permutation indeterminacy is solved using an approach based on the Hungarian algorithm that solves an Assignment Problem and an algorithm of Dynamic Programming. To obtain the distances in the Assignment Problem, a Kullback-Leibler divergence is adopted. The results of the experiments, performed using both synthetic and benchmark data, allows us to conclude that the approach presents a good performance and permits to obtain a clear separation of the signals also when they are more than two.
\end{abstract}

Mathematics Subject Classification: Information and communication, Fourier analysis.

Keywords: Blind Source Separation, Convolutive Mixtures, Fixed-Point Algorithm, Negentropy Information, Dynamic Programming. 


\section{Introduction}

The separation of convolutive mixtures of statistically independent sources is a fundamental problem in signal processing [6]. Blind Source Separation (BSS) consists in recovering statistically independent signals from the observations recorded by several sensors. Many Independent Component Analysis (ICA) algorithms have been proposed to solve BSS supposing that the observations are instantaneous mixtures of the sources [11], [9], [3], [1]. In real world situations, we observe convolutive mixtures of the sources and not instantaneous mixtures. Several methods have been proposed to solve the problem of separation of convolutive mixtures in time domain [3] [25] or in frequency domain [22], [16], [8]. The methods in time domain are limited [14] and computationally expensive. However, the main limitation of the apprHungarian algorithmoaches in frequency domain is the large number of instantaneous mixtures that must be separated in order to achieve a good performance. In addition, it is not easy to remove the permutation indeterminacies that appear from different frequency bins.

In this work, we use the fixed-point algorithm [9] in complex domain to obtain the separation of the convolved mixtures in frequency domain. To solve the amplitude dilation problem we propose a method based on a previous approach introduced by N. Murata et al. ([18]). To solve the permutation problem, we introduce a method based on an algorithm that solves an Assignment Problem and a Dynamic Programming algorithm [5]. In the Assignment Problem, to evaluate the distance between bins, we propose to use the Kullback-Leibler divergence [5]. We make different tests on synthetic and benchmark data to show the performance of our approach to recognize the permutations. Moreover, we compare our results with those obtained by some methods known in literature.

In section 2, we introduce the problem of BSS for convolutive mixtures. In section 3, we overview previous works with convolutive mixtures in frequency domain. In this section, we also introduce the fixed-point algorithm in complex domain (section 3.1), the Short Time Fourier Transform (section 3.2), some algorithms known in literature to achieve the separation (section 3.4) and some algorithms proposed to solve the amplitude and permutation indeterminacies (section 3.5). In section 4, we introduce the Assignment Problem (section 4.1), the Hungarian Algorithm (section 4.2) and the APDP approach (section 4.3). Finally, in section 5, we show the experimental results.

\section{The Convolutive BSS Problem}

In real world applications of ICA, some kind of convolution takes place simultaneously with the linear mixing. In fact, the source signals have different 
time delays in each observed signal due to the finite propagation speed in the medium. Moreover, each observed signal may contain time-delayed versions of the same source due to multipath propagation caused typically by reverberations from some obstacles. So, we observe convolutive mixtures and not instantaneous mixtures.

BSS of convolutive mixtures is basically a combination of standard instantaneous linear BSS and blind deconvolution. In the convolutive mixture model, each element of the mixing matrix $\mathbf{A}$ in the model $\mathbf{x}(t)=\mathbf{A s}(t)$ is a filter instead of a scalar. Written out for each mixture, the data model for convolutive mixtures is given by

$$
x_{i}(t)=\sum_{j=1}^{n} \sum_{k} a_{i k j} s_{j}(t-k) \quad \text { for } i=1, \ldots, n
$$

This is a FIR filter model, where each FIR filter is defined by the coefficients $a_{i k j}$. Usually these coefficients are assumed to be time-independent constants, and the number of terms over which the convolution index $k$ runs is finite.

To invert the convolutive mixtures in equation 1 , a set of similar FIR filters is typically used:

$$
y_{i}(t)=\sum_{j=1}^{n} \sum_{k} w_{i k j} x_{j}(t-k) \quad \text { for } i=1, \ldots, n
$$

The output signals $y_{1}(t), \ldots, y_{n}(t)$ of the separating system are estimates of the source signals $s_{1}(t), \ldots, s_{n}(t)$ at discrete time $t$. The $w_{i k j}$ 's are the coefficients of the FIR filters of the separating system. The FIR filters used in separation can be either causal or noncausal depending on the method. The number of coefficients in each separating filter must sometimes be very large for achieving sufficient inversion accuracy. Instead of the feedforward FIR structure, feedback (IIR type) filters have sometimes been used for separating convolutive mixtures.

\section{$3 \quad$ BSS in Time and Frequency Domains}

The problem of BSS for convolutive mixtures can be solved by extending ICA estimation criteria and algorithms developed for instantaneous mixtures. There are mainly two different approaches to solve the convolutive BSS problem. The main feature of the first approach is to work in the time domain $[3,1,25,14]$ and to obtain the separation of the sources estimating the coefficients $w_{i k j}$ (in (2)) of the FIR filters. We note that working in the time domain has the disadvantage of being rather computational expensive, due to calculating many convolutions. Other approaches suggested moving to the frequency domain in order to transform the convolution into multiplication and 
to apply ICA methods for instantaneous mixtures. Usually, the transformation used to pass in frequency domain is the Short Time Fourier Transform (STFT) $[19,20]$. We see some proposed algorithms, too. Finally, we see some methods known in literature to solve the problem of the permutation indeterminacy and amplitude indeterminacy. Remarking that the algorithm that we use to obtain the separation in complex domain is FastICA, in the following section, we show the features of this approach.

\subsection{Fixed-Point Algorithm in Complex Domain}

The fixed-point algorithm of complex signals requires a preliminary whitening of the data [4]. The one-unit learning rule is

$$
\begin{gathered}
\mathbf{w}^{+}=\underset{\left.\left|\mathbf{w}^{\mathrm{H}} \mathbf{x}\right|^{2} g^{\prime}\left(\left|\mathbf{w}^{\mathrm{H}} \mathbf{x}\right|^{2}\right)\right\} \mathbf{w}}{E\left\{\left.\mathbf{w}^{\mathrm{H}} \mathbf{x}\right|^{2}\right\}-E\left\{g\left(\left|\mathbf{w}^{\mathrm{H}} \mathbf{x}\right|^{2}\right)+\right.} \\
\mathbf{w}_{\text {new }}=\frac{\mathbf{w}^{+}}{\left\|\mathbf{w}^{+}\right\|} .
\end{gathered}
$$

In $(3), g(\mathbf{u})$ is the activation function. Different choices have been suggested $[4]$.

The one-unit algorithm can be extended to the estimation of the whole ICA transformation $\mathbf{s}(t)=\mathbf{W}^{\mathrm{T}} \mathbf{x}(t)$. To prevent the algorithm from converging to the same maxima, it is necessary to decorrelate the outputs of the network after every iteration. This has been accomplished using a deflation scheme based on Gram-Schmidt-like decorrelation [4].

We decide to use FastICA because it provides fast convergence and great separation quality.

\subsection{Short-Time Fourier Transform}

The first step of the method consists in applying the STFT to moving windows of the observed signals considering that the signals are stationary on short time-scale. This simplifies the problem considerably.

We split each observed signal $x_{i}(t)$ in $R$ overlapped frames of $K$ points, i.e. $\mathbf{x}_{i}\left(t_{r}\right)=\left[x_{i}\left(t_{r}\right), x_{i}\left(t_{r}+1\right), \ldots, x_{i}\left(t_{r}+K-1\right)\right]^{\mathrm{T}}$ where $t_{r}=r T, r=0, \ldots, R-1$ and $T$ is the time advance from one frame to the next one. Subsequently, we compute the $L$-point $(L \geq K)$ Discrete Fourier Transform of each frame obtaining [23, 19]

$$
X_{i}\left[\omega_{k}, t_{r}\right]=\sum_{m=0}^{K-1} x_{i}\left(t_{r}+m\right) e^{j \omega_{k} m} w(m)
$$


where $k=0, \ldots, L-1, \omega_{k}=2 \pi k / L$ denotes the frequency bin and $w(m)$ is the window (Hamming in our case). The STFT bin index is $k$.

\subsection{Frequency Domain Problem}

Applying the Fourier Transform techniques, the convolutions become products between Fourier transforms in frequency domain. In fact, in frequency domain the data model of (1) becomes

$$
X_{i}(\omega)=\sum_{j=1}^{m} A_{i j}(\omega) S_{j}(\omega), \text { for } i=1, \ldots, n
$$

where $X_{i}(\omega), S_{j}(\omega)$ and $A_{i j}(\omega)$ are the Fourier transforms of $x_{i}(t), s_{j}(t)$ and $a_{i j}(t)$, respectively.

Comparing (1) with (6), we note that the convolutive mixture problem is transformed into subproblems of instantaneous BSS/ICA models at each frequency. In this way, we can derive an algorithm for convolutive mixtures using a model for instantaneous mixtures. Using the STFT, the dependency of $X_{i}(\omega, t)$ on $\omega$ can be simplified dividing the values of $\omega$ into a fixed number of frequency bins (see equation 5). For every frequency bin, we have, then, a number of observations of $X_{i}(\omega, t)$, and we can estimate for each frequency bin the ICA models in complex domain.

\subsection{Frequency Domain Algorithms}

Smaragdis [22] proposed to apply the STFT to the convolutive mixtures. Then, to achieve separation from convolved mixtures, he applied to each frequency track, obtained from the STFT, the extension of the natural gradient ICA model [1] to complex source separation. Another algorithm, based on natural gradient in frequency domain, was that developed by Lee et al. [14]. The learning rules for such a system can be formulated using the FIR polynomial matrix algebra as described by Lambert [13]. Lee et al. formulated the infomax and natural gradient infomax rules in the frequency domain. Another approach was that developed by Mitianoudis and Davies [17]. They suggested to apply STFT on the observation signals. After this step, they estimated the unmixing matrix for every frequency bin using a fixed-point algorithm in complex domain (see 3.1).

However, one of the problems with these Fourier approaches is the indeterminacy of scaling and permutation. Scaling indeterminacy means that the scaling in each frequency band can be different. This leads to spectral deformations of the original sources. Permutation indeterminacy is a more difficult problem. It is essential to keep the same permutation to avoid that the signal sources have mixed frequency content. 


\subsection{Permutation and Amplitude Indeterminacies}

In the following, we show some algorithms proposed to solve the dilation and permutation indeterminacies.

\subsubsection{Influence Factor}

In the approach proposed by Smaragdis [22], the scaling problem is solved normalizing the unmixing matrices. For the permutation problem, he noted that a careful selection of the adaption parameters in addition to decaying learning rate and momentum are very helpful. In order to enforce the permutation, he used an influence factor to update each bin (IF approach). This approach is heuristic and, in some cases, it does not achieve the perfect solution.

\subsubsection{Unmixing filter}

Parra et al. [21] also worked in the frequency domain using non-stationarity to perform separation. They proposed to impose a constraint on the unmixing filter length $q$. This was achieved applying a projection operator $P$ to the filter estimates at each iteration, where $P=F Z F^{-1}$, where $F$ is the Fourier transform and $Z$ is a diagonal operator that projects on the first $q$ terms. Moreover, the heuristic adaptive solution of the previous section can be interpreted as placing weakly coupled priors on the unmixing matrix [17]. This imposes some weak smoothness constraint across frequencies, which should have an effect to the coupling, similar to that proposed by Parra et al. [21].

\subsubsection{Cross-Correlation and Cross-Cumulant}

Another recent work, was presented by Dapena et al. [8]. To solve the permutation indeterminacy, they proposed an approach based on the clustering of the outputs according to their cross-correlation (CC). The CC of two outputs was described by

$$
E\left[y_{i}[k-1] y_{l}^{*}[k]\right] .
$$

In this equation, $i, l=1, \ldots, m$ where $m$ is the total number of sources, $k=1, \ldots, L-1$ and $y_{i}[k]$ is the $i$-th source extracted at the $k$-th frequency bin. The authors normalized the sources so that $E\left[y_{i}[k] y_{i}^{*}[k]\right]=1$. To determine the $i$-th cluster $(i, l=1, \ldots, m)$ of the outputs corresponding to the same source, they used

$$
\begin{gathered}
U_{i}=\left\{u_{i}[0]=y_{i}[0], u_{i}[1]=\max _{y_{l}[1]}\left|E\left[y_{i}[0] y_{l}^{*}[1]\right]\right| \quad, \ldots,\right. \\
\left.u_{i}[L-1]=\max _{y_{l}[L-1]}\left|E\left[y_{i}[0] y_{l}^{*}[L-1]\right]\right|\right\}
\end{gathered}
$$


Considering the sources temporally white and stationary, it has been proved that this criteria can be used when $\widehat{\mathbf{f}}_{k}^{H} \widehat{\mathbf{f}}_{0}^{H} \neq 0$, for $k=1, \ldots, L-1$ where $\widehat{\mathbf{f}}_{k}$ $=\left[1, \ldots, e^{j 2 \pi k(F-1) / L}, \ldots, e^{j 2 \pi k(L-1) / L}\right]^{T}$, where $L$ is the DFT length and $F$ the order of the FIR filter. To solve the amplitude indeterminacy, the authors proposed to force all the outputs $u_{i}[k]$ into a set $U_{i}$ to have the same amplitude of $u_{i}[0]$.

In [15], the criterion proposed to solve the permutation was based on the spectral smoothness measured in terms of the dependencies between different frequency bands of the spectrogram by means of fourth order cross-cumulants.

\subsubsection{Splitting and Cross-Coherence}

To solve the ambiguity of permutation and dilation, a different approach was introduced in [18]. The authors applied the STFT to the input signals obtaining for the $j$-th frequency bin a term $X_{j}\left(\omega, t_{s}\right)$ depending on time. After the use of the Molgedey-Schuster' method [18], they obtained, for each frequency $\omega$, an estimated time sequence whose components were mutually independent

$$
u_{j}\left(\omega, t_{s}\right)=B(\omega) X_{j}\left(\omega, t_{s}\right)
$$

To solve the permutation and dilation problem, it was proposed to disassemble the spectrograms exploiting the independent components at each frequency channel. Split spectrograms were defined by

$$
\widehat{v}_{\omega}\left(t_{s} ; i\right)=B(\omega)^{-1}\left(\begin{array}{c}
0 \\
\vdots \\
u_{j}^{i}\left(\omega, t_{s}\right) \\
\vdots \\
0
\end{array}\right)
$$

where index $i$ denotes the dependence of the spectograms at $\omega$ on the $i$-th independent component of $u_{j}^{i}\left(\omega, t_{s}\right)$. In order to obtain $\widehat{v}_{\omega}\left(t_{s} ; i\right), B(\omega)$ and $B(\omega)^{-1}$ were applied so that to eliminate the ambiguity of dilation in $\widehat{v}_{\omega}\left(t_{s} ; i\right)$. Murata et al. [18] utilized the non-stationarity of the source signals to obtain the sorting of the permutations. If the split band-passed signals $\widehat{v}_{\omega}\left(t_{s} ; i\right)$ are originated from the same source signal, then it is natural to assume that they are under the influence of similar modulations in amplitude. The permutation was solved by sorting the coherence of the envelops obtained with an $\varepsilon$ operator [18]. Sorting was determined with the correlation between the envelops of band-passed signals. The number of independent signals obtained for each separated source was equal to the cardinality of the signals analyzed $\mathbf{x}(t)$. 


\section{APDP Approach}

In this section, we propose a new approach to solve the permutation indeterminacy based on an algorithm that solves an Assignment Problem (AP) and an algorithm of Dynamic Programming [5]. Here, we consider two adjacent bins as source and destination nodes in an Assignment Problem. The aim is then to minimize the sum of the costs between the nodes. To solve the Assignment Problem, we use the Hungarian algorithm (section 4.2). Considering that in some situations we need a global information to obtain the matching, we couple the Hungarian algorithm and the Dynamic Programming algorithm (section 4.3). This new strategy presents a better performance than the others presented in the paper. This can be derived from the experiments on synthetic and benchmark data (section 5) that we present in the following section.

\subsection{Assignment Problem}

In this section the concept of optimal assignment is reviewed, and the metric properties of this assignment are investigated. It is assumed that two sets of features of equal cardinality are provided, and that a weighting (measure of distance) between each feature in one set to all the features in the other set is given. The value of the sum of all weights between corresponding features is denoted here as $\delta(A, B)$ where $A$ and $B$ denote the two sets. In our case, the distance $\delta(A, B)$ between two sets $A$ and $B$ is given by an optimal assignment of each element $a_{i}$ in $A$ to an element $b_{j}$ in $B, f: A \rightarrow B$, such that the sum of the distances for the assignment is minimized.

Equivalently, this can be represented as a graph theory problem in which sets $A$ and $B$ correspond to the two sets of a bipartite graph. A graph $G$ is called bipartite if its vertex set can be divided into two independent sets $A, B$ [7]. Therefore, no edge in the graph joins two vertices in the same subset. A matching $M$ in a graph $G$ is defined as a set of pairwise disjoint edges. The task then is to find a perfect matching (i.e., a one-to-one and onto matching) in a weighted bipartite graph [2] [7] $G=(A, B)$, such that the sum of the weights of the matching is minimized. The weights correspond to the distance between two elements.

Let $x_{i j}$ be a variable which is 1 if feature $a_{i}$ in the present set maps to feature $b_{j}$ in the new feature set and 0 otherwise and $d_{i j}=\delta_{E}\left(a_{i}, b_{i}\right)$ is the distance between features $a_{i}$ and $b_{j}$. An arbitrary assignment will have an associated cost function

$$
f(A, B)=\sum_{i=1}^{n} \sum_{j=1}^{n} d_{i j} x_{i j}
$$


A mathematical model for the assignment problem is given by the following

$$
\begin{array}{ccc}
\text { Minimize } & f(A, B) & \\
\text { Subject to } & \sum_{j=1}^{n} x_{i j}=1 & i=1, \ldots, n \\
& -\sum_{i=1}^{n} x_{i j}=-1 & j=1, \ldots, n \\
& x_{i j}=0 \text { or } 1 \quad i, j=1, \ldots, n
\end{array}
$$

The constraints ensure that the mapping is a bijection. A measure of distance between the two sets is defined as:

$$
\delta(A, B)=\min _{\Pi_{n}} f(A, B)
$$

where $\Pi_{n}$ is the set of all possible matchings. In matrix form, the assignment problem becomes as follows:

$$
\begin{gathered}
\text { Minimize } \quad \mathbf{d} \mathbf{x} \\
\text { Subject to } \quad \mathbf{A} \mathbf{x}=\mathbf{b} \\
x_{i j}=0 \text { or } 1 \quad i, j=1, \ldots, n
\end{gathered}
$$

where $\mathbf{x}=\left[x_{11}, \ldots, x_{1 n}, \ldots, x_{n 1}, \ldots, x_{n n}\right]^{T}, \mathbf{A}$ is a $2 n \times n^{2}$ matrix whose $(i, j)$ column is $\mathbf{a}_{i j}=\mathbf{e}_{i}-\mathbf{e}_{n+j}$ for $i, j=1,2, \ldots, n$ and $\mathbf{b}=[\mathbf{1},-\mathbf{1}]^{T}$, where $\mathbf{1}$ is a vector of $n$ ones and $\mathbf{e}_{k}$ is the canonical base vector having 1 at $k$-th position and zero anywhere. We note that since the AP is a special case of the Transportation Problem [2] we could apply the same algorithms to solve the AP. In the following, we focus our attention on the Hungarian algorithm (HA) for optimal assignment. We note that a different polynomial-time algorithm can be used based on solving a succession of shortest path problems [2]. In this case, we could use a dynamic programming algorithm. We observe that the assignment polytope has $n$ ! extreme points. In the following, we introduce the Hungarian Algorithm that solve the AP problem with a complexity of $O\left(n^{3}\right)$ where $n$ is the number of points.

\subsection{Hungarian Algorithm}

Now we summarize the steps to calculate the optimal AP using the HA algorithm

Step 1 - For each point $i$, find its distance $d(i, j)$ from point $j$. Construct a $n \times n$ matrix $\mathbf{D}$ with $d_{i j} \in \mathbf{D}$. For each row $q$, let $k_{q}$ the minimum element, subtract $k_{q}$ from each element of $q$. 
For each column $p$, let $l_{p}$ be the minimum element, subtract $l_{p}$ from each element of $p$.

Step 2 - Find a minimum set of lines covering the independent 0's. If the number of lines is equal to $n$, go to Step 4, else go to Step 3.

Step 3 -Find the smallest uncovered element $p$. Subtract $p$ from each uncovered element. Add $p$ to each element lying at the intersection of two lines. Go to Step 2.

Step 4 -Find an independent set of 0's. Sum $d(i, j)$ 's corresponding to the $n$ independent 0 's. Output this as the value of $\delta$ (equation 11).

\subsection{APDP algorithm}

In this section, we show the approach to solve the permutation indeterminacy based on the HA that solves the Assignment Problem and on a Dynamic Programming (DP) algorithm (following APDP approach). We note that we can consider the outputs of two adjacent bins as sources in $A$ and destinations in $B$ in an AP. To this problem can be associated an arbitrary assignment that has an associated cost function as in (11). Now, the task is to find a perfect matching in a weighted bipartite graph $G=(A, B)$, such that the sum of the weights of the matching is minimized. Let $x_{i j}$ be a variable which is 1 if feature $a_{i}$ in the present set maps to feature $b_{j}$ in the new feature set and 0 otherwise and $d_{i j}=\delta_{E}\left(a_{i}, b_{i}\right)$ is the distance between features $a_{i}$ and $b_{j}$. We consider as distance $d_{i j}$ the Kullback-Leibler (KL) divergence.

The KL divergence is defined between two discrete $n$-dimensional probability density functions $\mathbf{p}=\left[p_{1} \ldots p_{n}\right]$ and $\mathbf{q}=\left[q_{1} \ldots q_{n}\right]$ as

$$
K L(\mathbf{p}, \mathbf{q})=\sum_{i=1}^{n} p_{i} \log \frac{p_{i}}{q_{i}}
$$

This divergence can be considered as a kind of distance between two probability densities, because it is always nonnegative, and zero if and only if the two distributions are equal.

We know that a single output of a frequency bin represents the Fast Fourier Transform at different frames.

In the following, we obtain good results defining the probability density $p$ as the normalized Power Spectrum Densities (PSD) of each signal.

In this way we have that for non-stationary source signals adjacent bins in the STFT have approximatively the same temporal features and the same amplitude. 
In details, we define the $j$-th component of the discrete distribution $\mathbf{p}_{k}^{i}=$ $\left[p_{k, 1}^{i} \ldots p_{k, R}^{i}\right]$ as

$$
p_{k, j}^{i}=\frac{\left|\mathbf{X}_{i}\left(\omega_{k}, j\right)\right|^{2}}{\sum_{s=1}^{R}\left|\mathbf{X}_{i}\left(\omega_{k}, s\right)\right|^{2}}
$$

where $\mathbf{X}_{i}\left(\omega_{k}, j\right)$ is the Fourier Transform of the $i$-th extracted source at the $j$-th frame of the $k$-th frequency bin and $R$ is the total number of frames.

However we have to note that in our approach any probability density and/or pre-processing could be used.

We also mark that the KL divergence is not a proper distance measure because it is not symmetric. In our experiments we also use a symmetric version of it [27].

In section 4.1, we have seen that the $\mathrm{HA}$ is a good candidate to solve the problem proposed in (11). We note that applying the AP we can obtain only a local information about signals in frequency domain because we are solving the problem only for adjacent bins. To achieve a global solution, we propose to use a DP algorithm.

DP is typically applied to optimization problems. In such problems there can be many possible solutions. Each solution has a value, and we wish to find a solution with the optimal (minimum or maximum) value. We call such a solution an optimal solution to the problem, as opposed to the optimal solution, since there may be several solutions that achieve the optimal value. The development of a DP algorithm can be broken into a sequence of four steps:

1. Characterize the structure of an optimal solution;

2. recursively define the value of an optimal solution in terms of the optimal solutions to subproblems;

3. compute the value of an optimal solution in a bottom-up fashion;

4. construct an optimal solution from computed information.

Our idea is to apply an HA to solve the AP between bins and the DP to search the optimal path between the bins.

We also note that adjacent bins have similar distributions. Then to prevent the matching between bins that have long distances and to assure an overall coverage of the bins, we add to our approach an $\alpha$ parameter that acts as a "loss factor". 
In details if we have $m$ source signals and $n$ frequency bins the recursive relation is

$$
\begin{array}{lll}
c[i]= & \min _{i-j \leq k<i} F(A, B) & \forall i>j \text { and } i<n \\
c[i]= & \min _{1 \leq k<i} F(A, B) & \forall i>1 \text { and } i \leq j \\
c[i]= & 0 & \text { if } k=1 \\
\text { Sub. to } & \sum_{s=1}^{m} x_{s t}=1 & s=1, \ldots, m \\
& -\sum_{s=1}^{m} x_{s t}=-1 & t=1, \ldots, m \\
& & s, t=1, \ldots, m
\end{array}
$$

where

$$
F(A, B)=\min \sum_{s=1}^{m} \sum_{t=1}^{m} x_{s t}\left(d_{s t}^{i, k}+\alpha c[k]\right)
$$

$d_{s t}^{i, k}$ is the distance (divergence in our case) between the $s$-th and the $t$-th estimated sources at the $i$-th and $k$-th bins, respectively, and $0<\alpha \leq 1$ is the loss factor. Since we need to search bins (or group of bins) where the separated sources have similar and noiseless distributions we also introduced a $j$ parameter that permits to define the size of a window where we can search for the optimal matches.

In other words in this approach we apply the HA between the $m$ separated signals of the $i$-th and the $k$-th bins, respectively. At this point $c[n]$ contains the minimum error of the best path between the bins. The path depends on the $j$ parameter and could not cover all the bins. In this case we use the minimum of the error at the generic $i$-th recursive relation to obtain the optimal permutation to the subproblem.

We also note that in the case $j=1$ the minimum of the overall error (optimal permutation) is contained in $c[n]$ and the path cover all the bins. We mark that in this way if we consider noiseless and well separated sources then the recursive relation achieve the optimal permutation problem.

The computational complexity of the algorithm is $O\left(\mathrm{njm}^{3}\right)$ where $j$ is the parameter in equation 17, and the spatial complexity is $O(n)$.

We note that the IF and CC algorithms permit to obtain a lower complexity, in both the cases, than our method. However we note that the first approach is a heuristic and in the second case we can obtain only a local matching. Moreover, we note that the complexities in Murata et al. approach are comparable with that obtained with our approach since we need to sort the coherence of the envelops of the separated signals. 
In the APDP approach to solve the ambiguity of the amplitude dilation we propose a simple modification of a previous method proposed by N. Murata et al. (section 3.5.4). In fact, since with this method we obtain for each separated signal a number of signals that is equal to the number of mixtures (see section 3.5.4), then we simply propose to obtain a mean of the signals for each output.

\section{$5 \quad$ Experimental Results}

In this section, we show some results obtained using the APDP to solve the permutation problem. We note that this approach is tested on synthetic data and on benchmark data.

In order to evaluate the performance of our approach and compare it with that of other methods, we use the criterion proposed in [12], [24]. Instead of using the Amari index, the authors of these papers judge the accuracy of the source estimates looking at the Mutual Information (MI) between the sources. If and only if the sources were estimated correctly, the MI is zero. The important advantage over the Amari index, is that this approach can also be used when the exact sources are not known. For further details see [12], [24].

Having a criterion to evaluate the performance, we have also checked that the best result for all the tests performed is obtained when the $\alpha$ parameter of the $\mathrm{DP}$ is chosen equal to $1 / 4$.

\subsection{Experimental results using simulated data}

The aim of the first test is to show how the APDP performs. We use two periodic signals: a Gaussian-modulated sinusoidal pulse and a Gaussian-noise (Fig. 1). We simulate the separation process of the ICA outputs, mixing the two spectrograms of the signals, with a percentage of permutation of $50 \%$. To simulate the loss of amplitude, we normalize each bin of the two spectrograms. In Fig. 2, we plot the signals obtained after the mixing process and the normalization of amplitude and their spectrograms. We apply different approaches to solve the permutation. In Fig. 3a-c, we show, respectively, the results of CC approach (section 3.5.3), the results obtained applying the approach described in section 3.5.4, and the ones after APDP approach. From this preliminary analysis, we note that the APDP performs better than the other approaches and permits to obtain a good performance to solve the permutation problem. In fact, in all the other cases, we have that the permutation is unsolved. Graphically, this can be seen from the spectrograms that contain information of the other signals. 


\subsection{Experimental results using T.W. Lee benchmark data}

In this section, we show the results obtained applying the APDP approach on the benchmark data available at [26].

The first set of data is made up of convolved mixtures (Fig. 4a) of two signals recorded in a normal office room by two microphones having a sampling rate of $16 \mathrm{kHz}$. The sources are the sound of a speaker saying the digits "one" to "ten" and the music coming from a cassette player. In Fig. 4b, we plot the results obtained after the separation using the algorithm proposed by T.W. Lee at al. (TWL approach) [14]. In Fig. 4c, we show the results obtained after the separation using FastICA algorithm in complex domain and the IF approach to solve the permutations (IF approach). In Fig. 4d, we plot the results obtained after the separation using the FastICA algorithm in complex domain and the CC approach (CC approach). In Fig. 4e, we show the results obtained after the separation using FastICA algorithm in complex domain and the APDP approach (APDP approach). We note that the APDP approach permits to obtain better results than the IF and CC approaches. Moreover, we have that using the APDP approach, the results are better than the ones obtained by TWL algorithm. This is evident comparing the performances in Tab. 1.

The second set of data available at [26] consists in two convolved mixtures (Fig. 5a) of two people speaking in a normal office room. The two microphones have a sampling rate of $16 \mathrm{kHz}$. One of the speakers says the digits "one" to "ten" in English, the other one the same digits in Spanish. In Fig. 5b, we plot the results obtained after the separation using the algorithm proposed by T.W. Lee at al. (TWL approach) [14]. In Fig. 5c, we show the results obtained after the separation using IF approach. In Fig. 5d, we plot the results obtained after $\mathrm{CC}$ approach. In Fig. 5e, we show the results obtained after the separation using APDP approach. We note that the APDP approach permits to obtain better results than the IF and CC approaches. Moreover, also in this case, the results obtaining using the APDP approach are better than the ones of TWL algorithm. This is clear comparing the performances in Tab. 1.

\subsection{Experimental results using Ikeda benchmark data}

We perform also another test on a set of data available at [28]. In this experiment, we want to separate the sounds of two male speakers recorded with a sampling rate of $16 \mathrm{KHz}$. The signals are the word "good morning" and a Japanese word "konbanwa". In Fig. 6a, we plot their convolved mixtures. In Fig. $6 \mathrm{~b}$, we show the results obtained using the approach proposed in [18] (section 3.5.4). In Fig. 6c, we plot the results obtained using the IF approach. 
In Fig. 6d, we show the results obtained by $\mathrm{CC}$ approach. In Fig. 6e, there are the results obtained using APDP approach. Also in this case, the results of our separation are good, better than the ones obtained by IF and CC approaches and very similar to those obtained by Ikeda method. This is evident comparing the performances in Tab. 2. Note that in this table we report four performance indexes for Ikeda approach since it gives four separated signals, two for each source. So we have an index for each of the four combinations of sources.

\subsection{Experimental results using Schobben benchmark data}

In the following, we present the results obtained on a more difficult benchmark data available at [29]. In the first experiment on this data, a male and a female speaker are speaking simultaneously and there is some background noise. The signals are sampled at $16 \mathrm{KHz}$ and are 10 seconds long. In Fig. 7a, the contribution of the sources to the microphones is plotted. In Fig. 7b and Fig. 7c, we show the results obtained after separation using Schobben and APDP approach, respectively. Also in this case, we obtain a good performance and listening the separated signals we note that they are clear. In particular, the male source is well separated. Our results are better than the ones obtained by Schobben. This is clear comparing the performances in Tab. 3 .

In the second experiment available at [29], the tracks are recorded in a room that is not completely anechoic, but it does have a very short reverberation time. This recording contains no noise. Two male speakers are recorded using two microphones. The original sources are not available at [29] as in the first experiment. In Fig. 8a, we show their convolved mixtures. In Fig. 8b and Fig. 8c, we plot the signals separated by Schobben and APDP approach, respectively. We have a clear separation better than the one obtained using the Schobben algorithm. This is evident comparing the performances in Tab. 3 .

\subsection{Experimental results using multichannel simulated data}

To conclude, we present the results of an experiment on simulated data. We create convolutive mixtures of four sources: a Gaussian-modulated sinusoidal pulse, a periodic signal, a chirp signal and gaussian noise. The mixing system $A_{i j}(z)$ of the $i$-th mixture and $j$ th source signal has the following algebraic form

$$
A_{i j}(z)=\frac{\sum_{k=0}^{M} b_{k}^{i j} z^{-k}}{\sum_{k=0}^{M} a_{k}^{i j} z^{-k}} .
$$


In our experiment, we consider $M=2, N=0, a_{k}^{i j}=1$ and $b_{k}^{i j} \epsilon[-1,1]$ randomly chosen. We plot the simulated sources and their convolutions in Fig. 9a-b, respectively. The separation obtained by APDP approach is very good (Fig. 9c). To the best of our knowledge, this is the first experiment with more than three sources presented in a paper.

\begin{tabular}{|c|c|c|}
\hline \multicolumn{3}{|c|}{ T.W. Lee benchmark data } \\
\hline \hline & speech-music & speech-speech \\
\hline TWL & 0.00300 & 0.6153 \\
\hline APDP & 0.00013 & 0.0660 \\
\hline
\end{tabular}

Table 1: Performance index obtained by TWL and APDP method for T.W. Lee benchmark data.

\begin{tabular}{|c|c|c||c||c|}
\hline \multicolumn{5}{|c|}{ Ikeda benchmark data } \\
\hline \hline Ikeda & 0.1176 & 0.1172 & 0.0902 & 0.0932 \\
\hline APDP & 0.1094 & & & \\
\hline
\end{tabular}

Table 2: Performance index obtained by Ikeda and APDP method for Ikeda benchmark data.

\begin{tabular}{|c|c|c|}
\hline \multicolumn{3}{|c|}{ Schobben benchmark data } \\
\hline \hline & female-male & male-male \\
\hline Schobben & 0.2419 & 0.3207 \\
\hline APDP & 0.0777 & 0.1437 \\
\hline
\end{tabular}

Table 3: Performance index obtained by Scobben and APDP method for Schobben benchmark data.

\section{Conclusion}

In this paper, we proposed an approach to solve the permutation indeterminacy in the separation for BSS of convolved mixtures in frequency domain. We used the fixed-point algorithm in complex domain to perform the separation of the signals for each frequency domain. To determine the frequency bins, we used a Short Time Fourier Transform on a set of fixed frames. To solve the ambiguity of the amplitude dilation, we proposed a simple approach deriving from a previous method proposed by N. Murata et al. (Section 3.5.4). The 

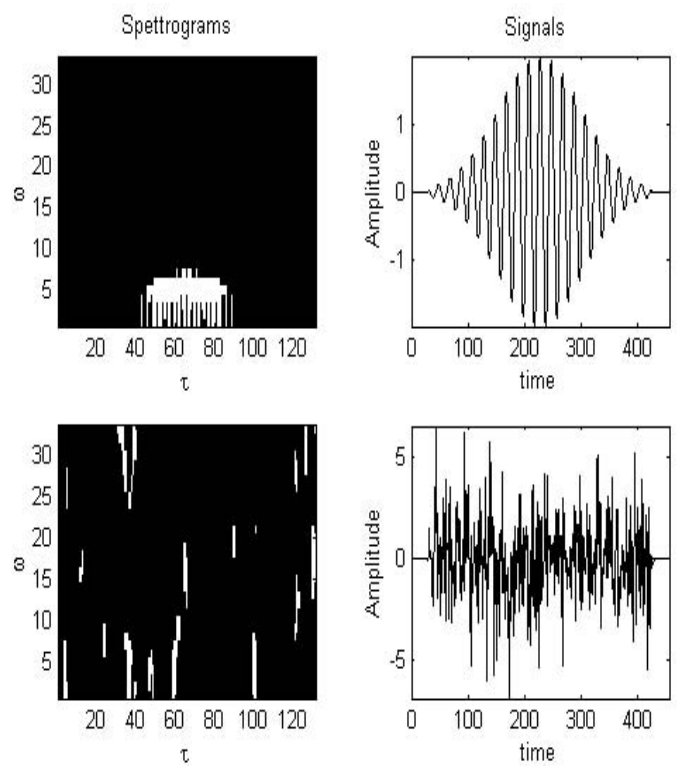

Figure 1: Spectrogram of Gaussian-modulated sinusoidal pulse (upper panel); spectrogram of a Gaussian noise (bottom panel)
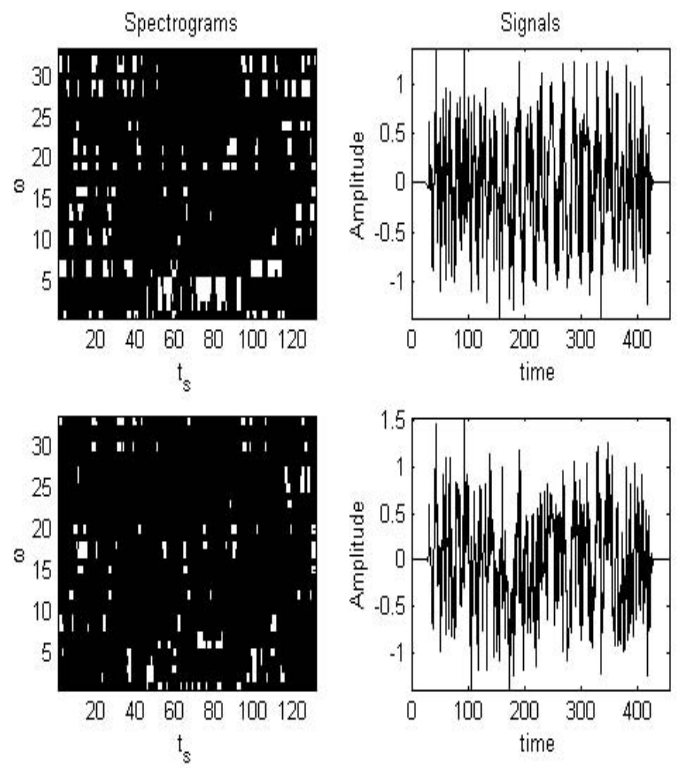

Figure 2: Spectrograms of the signals of the previous picture after the mixing process and the normalization of amplitude 

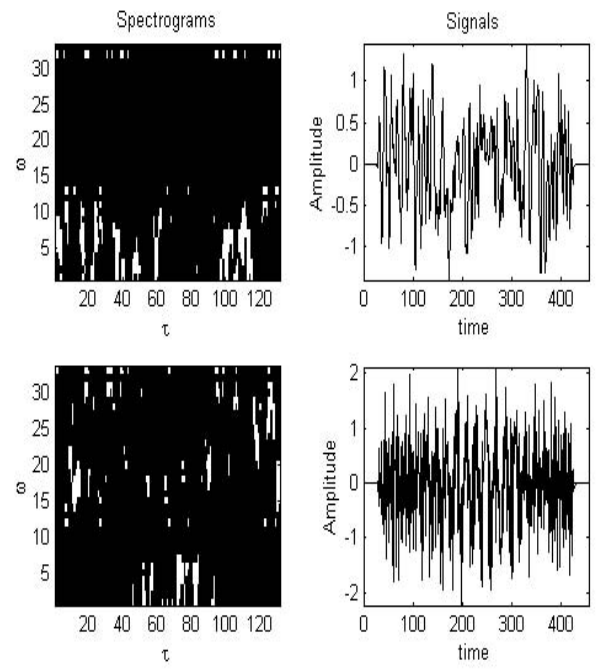

a)
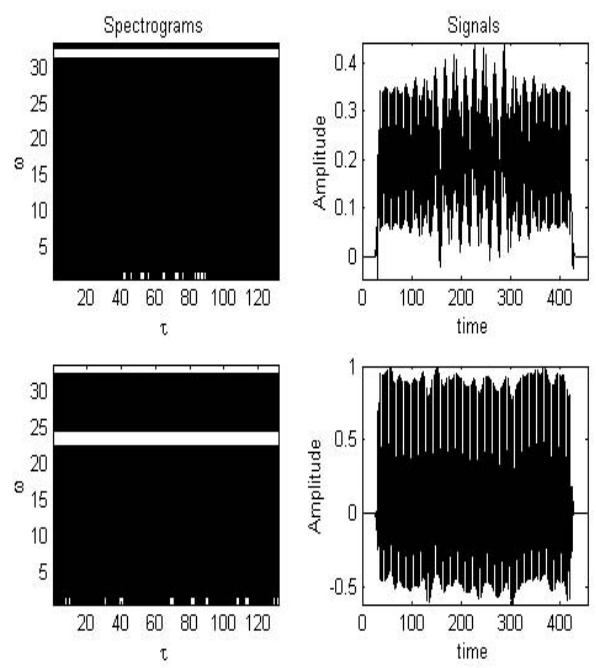

b)
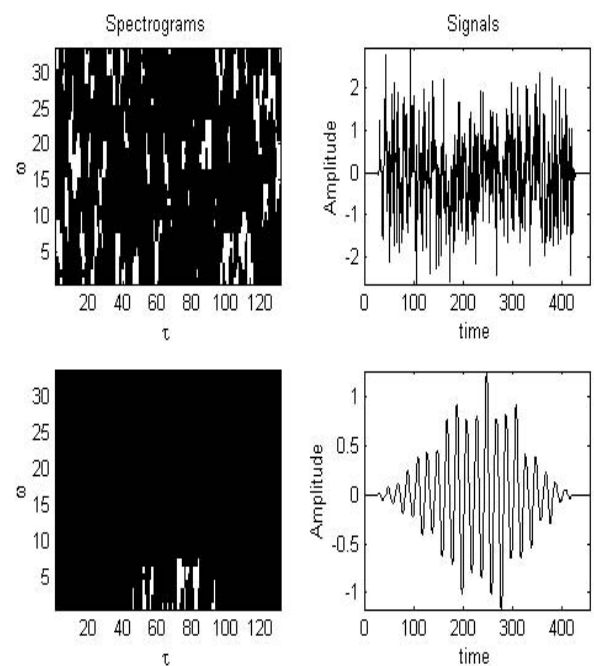

c)

Figure 3: a) Spectrograms of the separated sources obtained by CC approach; b) spectrograms of the separated sources obtained by IF approach; c) spectrograms of the separated sources obtained by APDP approach; 

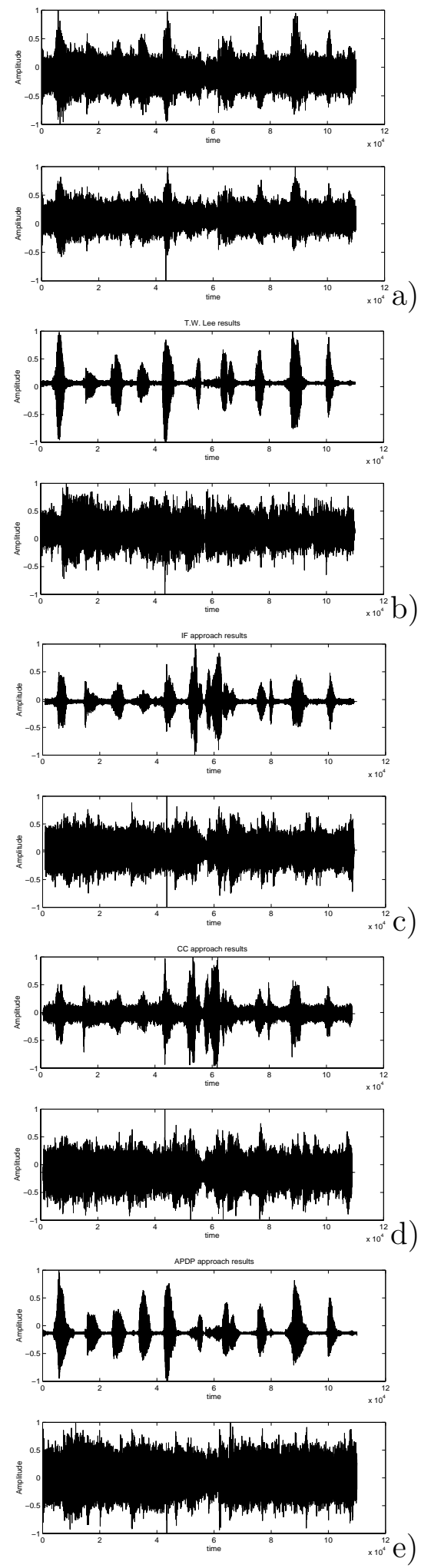

Figure 4: a) Speech-Music convolutive mixtures; b) separated sources using TWL method; c) separated sources using IF approach; d) separated sources using CC approach; e) separated sources using APDP method 

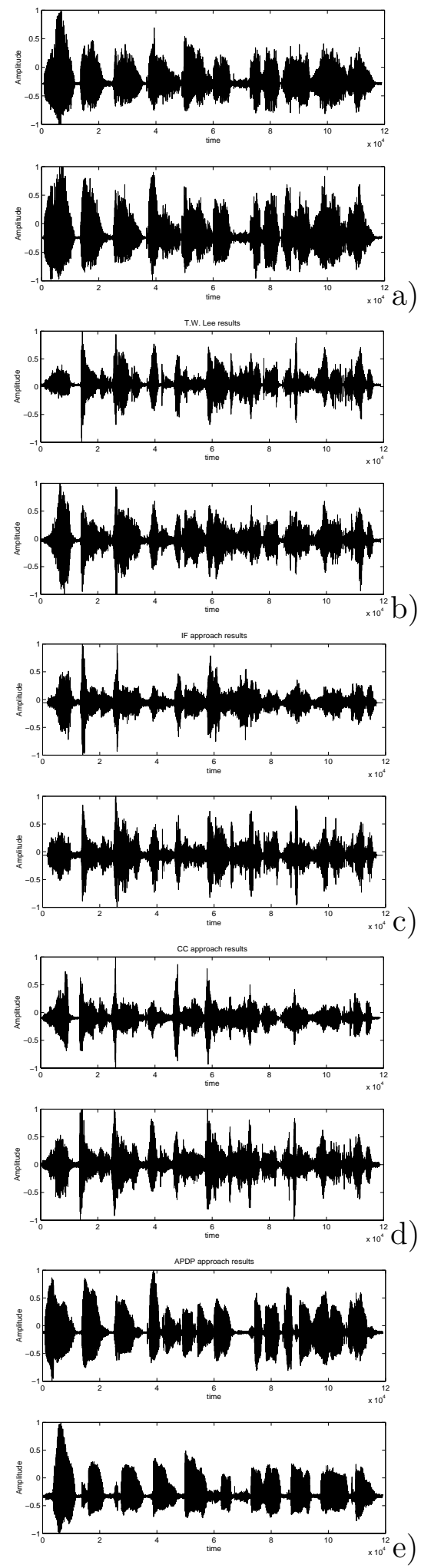

Figure 5: a) Speech-Speech convolutive mixtures; b) estimated sources by TWL method; c) estimated sources by IF approach; d) estimated sources by CC approach; e) estimated sources by APDP method 

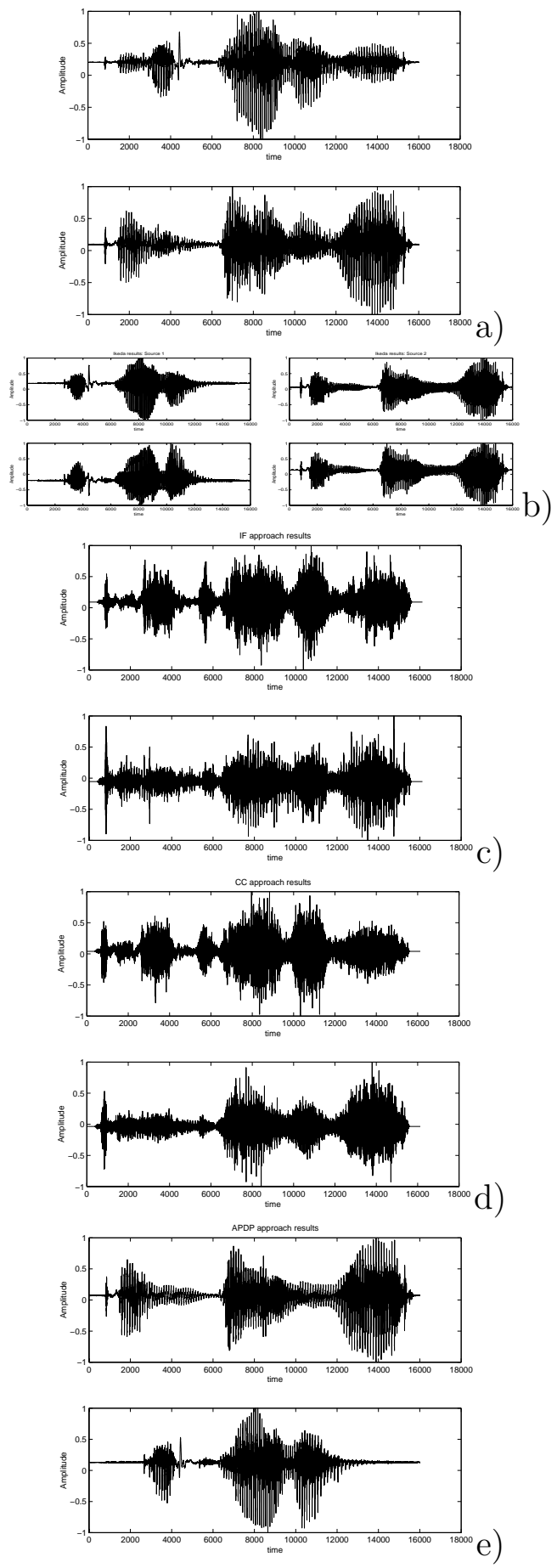

Figure 6: a) Speech-Speech convolutive mixtures; b) separated sources using Ikeda method (source 1 is in left panel, source 2 is in right panel; c) separated sources using IF approach; d) separated sources using CC approach; e) separated sources using APDP approach 

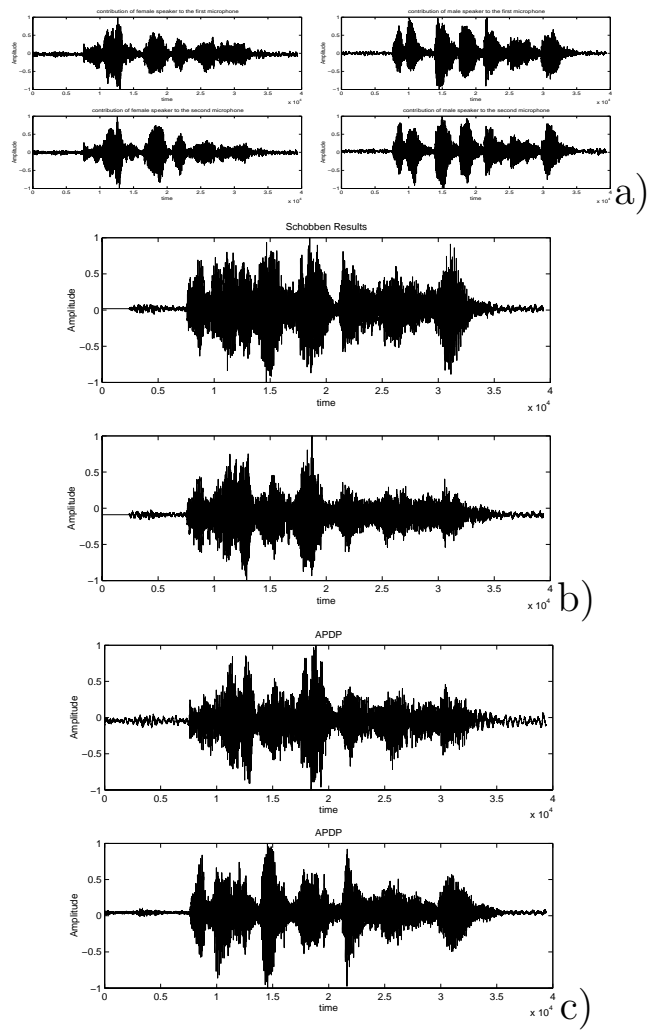

Figure 7: a) Contribution of female speaker to microphones (left panel); contribution of male speaker to microphones (right panel); b) estimated sources by Schobben approach; c) estimated sources by APDP approach 

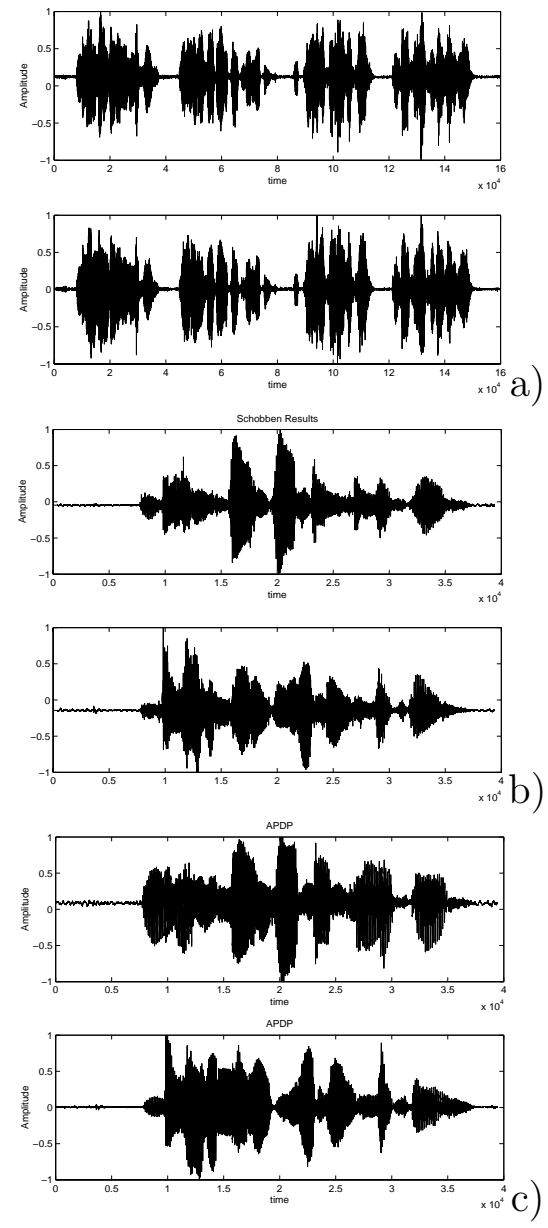

Figure 8: a) Speech-Speech convolutive mixtures; b) estimated sources by Schobben approach; c) estimated sources by APDP approach b) 

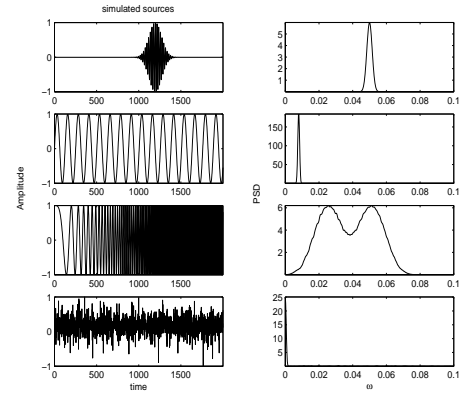

a)
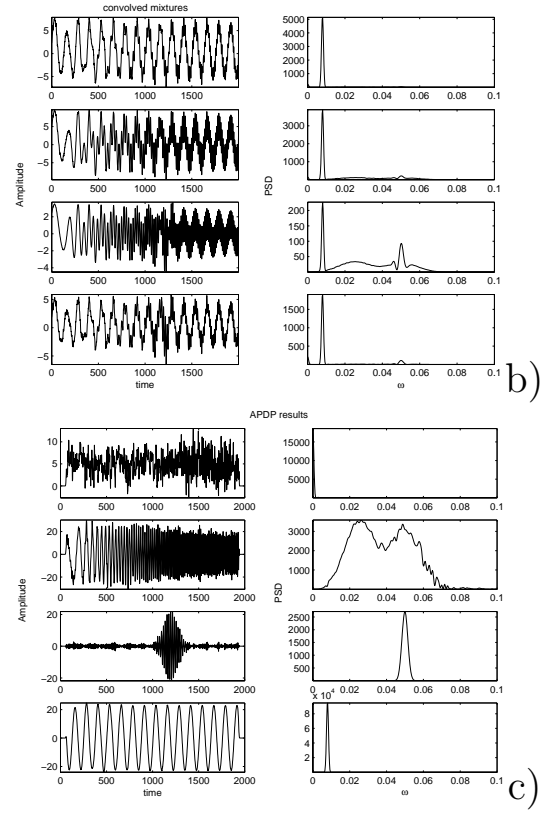

Figure 9: a) the four simulated sources: a Gaussian-modulated sinusoidal pulse, a periodic signal, a chirp signals and gaussian noise; b) their convolutions; c) estimated sources by APDP approach 
proposed approach is based both on Hungarian algorithm that solves an Assignment Problem and on an algorithm of Dynamic Programming. From the experimental results, we have seen that this approach presents a good performance and permits to obtain a clear matching also when the sources are more than two.

We note that the experiments were made comparing the new method with the others most cited in literature on their own benchmarks. It was not possible to compare all the methods with all data since the other algorithms are not available either in internet or under request.

\section{Acknowledgment}

This work has been partially supported by CNR-ASI and MIUR.

\section{References}

[1] S.-I. Amari, A. Cichocki, H.H. Yang. "A new learning algorithm for blind source separation", Advances in Neural Information Processing Systems 8, pp. 757 - 763, MIT Press, 1996.

[2] M.S. Bazaraa, J.J. Jarvis, H.D. Sherali. Linear Programming and Network Flows, John Wiley \& Sons, II edition, 1990.

[3] A. Bell, T. Sejnowski. "An Information-maximization approach to blind separation and Blind Deconvolution". Neural Computation, 7:1129-1159, 1995.

[4] E. Bingham, A. Hyvärinen. "A fast fixed-point algorithm for independent component analysis of complex-valued signals", International Journal of Neural Systems, 10(1):1-8, 2000.

[5] A. Ciaramella, R. Tagliaferri. "Amplitude and Permutation Indeterminancies in Frequency Domain Convolved ICA", to appear in proceedings of IJCNN 2003, Porylandi, Oregon, USA.

[6] A. Cichocki, S.I. Amari. Adaptive Blind Signal and Image Processing, Wiley , 2003.

[7] T.H. Cormen, C.E. Leiserson, R.L. Rivest. Introduction to Algorithms, MIT Press, 1990.

[8] A. Dapena, M.F. Bugallo and L. Castedo. "Separation of convolutive mixtures of temporally-white signals: a novel Frequency-Domain Approach". 
In Proc. 3rd International Workshop on Independent Component Analysis and Blind Source Separation, San Diego, California, pp. 179-184, 2001.

[9] A. Hyvärinen, E. Oja. "A fast fixed-point algorithm for independent component analysis". Neural Computation, 9(7):1483-1492, 1997.

[10] A. Hyvärinen. "Fast and robust fixed-point algorithms for independent component analysis". IEEE Trans. On Neural Networks, 10(3):626-634, 1999 .

[11] A. Hyvärinen, J. Karhunen, E. Oja. Independent Component Analysis", John Wiley \& Sons, 2001.

[12] A. Kraskov, H. Stögbauer, P. Grassberger. "Estimating Mutual Information". To appear on Physical Review E 69, 066138 , 2004. http://arxiv.org/abs/q-bio/0311037

[13] R. H. Lambert. Multichannel Blind Deconvolution: FIR Matrix Algebra and Separation of Multipath Mixtures, PhD thesis, Univ. of Southern California, 1996.

[14] T.-W. Lee, M. Girolami and R. Lambert. "Blind separation of delayed and convolved sources". In Advances in Neural Information Processing Systems, volume 9, pp. 758-764, MIT Press, 1997.

[15] C. Mejuto, A. Dapena, L. Castedo. "Frequency-Domain Infomax for Blind Source Separation of Convolutive Mixtures". Proc. ICA 2000, pp. 315-320, Helsinki, 2000.

[16] N. Mitianoudis and M. Davies. "New fixed-point ICA algorithm for convolved mixtures ". In Proc. 3rd International Workshop on Independent Component Analysis and Blind Source Separation, San Diego, California, pp. 633-638, 2001.

[17] N. Mitianoudis, M. Davis. "Audio Source Separation of Convolutive mixtures". IEEE Trans. on Speech and Audio Processing, to appear.

[18] N. Murata, S. Ikeda, A. Ziehe. "An approach to Blind Source Separation based on Temporal Structure of Speech Signals". Neurocomputing, 41:1-24, 2001.

[19] A. V. Oppenheim, R. W. Shafer. Disrete-Time Signal Processing, Prentice-Hall,Inc., 1989.

[20] A. Papoulis. Probability, Random Variables, and Stochastic Processes, McGraw?Hill, 3rd edition, 1991. 
[21] L. Parra, C. Spence. "Convolutive blind source separation of nonstationary sources". IEEE Trans. on Speech and Audio Processing, pp. 320-327, May 2000.

[22] P. Smaragdis. "Blind separation of convolved mixtures in frequency domain". Neurocomputing, 22:21-34, 1998.

[23] J. O. Smith III, X. Serra. "PARSHAL: An Analysis/Synthesis Program for Non-Harmonic Sounds Based on a Sinusoidal Representation". CCRMA STAN-M-43, Proc. Int. Computer Music Conf. (ICMC-87), ChampaignUrbana, 1987.

[24] H. Stögbauer, A. Kraskov, S.A. Astakhov, P. Grassberger. "Least Dependent Component Analysis based on Mutual Information". Submitted to Physical Review E, 2004. http://arXiv.org/abs/physics/0405044

[25] K. Torkkola. "Blind separation of convolved sources based on information maximization". In Proc. IEEE Workshop on Neural Networks and Signal Processing (NNSP'96), Kyoto, Japan, pp. 423-432, 1996.

[26] http://inc2.ucsd.edu/ tewon/ica_cnl.html

[27] http://www.ai.mit.edu/courses/6.891-f99/ps/5/matlab/BNT/general/

[28] http://www.ism.ac.jp/ shiro/research/index.html

[29] http://www2.ele.tue.nl/ica99/realworld.html

Received: September 23, 2005 